Open Access

\title{
Numerical investigation of an active TPS for a wing leading edge exposed to high temperature air behind a strong bow shock wave
}

\author{
Taku Ohwada*, Tomonori Shimada and Takuma Kato
}

* Correspondence:

ohwada@kuaero.kyoto-u.ac.jp

Department of Aeronautics and Astronautics, Graduate School of Engineering, Kyoto University, Kyoto 615-8530, Japan

\begin{abstract}
An array of subsonic counter-flow jets is studied as an active thermal protection system (TPS) for wing leading edges of hypersonic vehicles. The performance is numerically estimated in the model case of a circular cylinder on the basis of the 2D compressible Navier-Stokes equations. In contrast to a single subsonic jet, an array of jets is robust against variation of the angle of attack; high cooling effectiveness is confirmed up to $5^{\circ}$ variation. The coolant gas (air) discharged from channels embedded in the cylinder covers over a wide range of the front surface of the cylinder. The feasibility of the active TPS is also discussed.
\end{abstract}

Keywords: Counter-flow jet, Hypersonic vehicle, Thermal protection system, Compressible Navier-Stokes, Shock capturing

\section{Introduction}

A hypersonic vehicle must bear harsh aerodynamic heating and the implementation of thermal protection system (TPS) is mandatory in particular around the nose cap and the wing leading edges. Re-usability, low-cost maintenance and life-elongation are now regarded as requisite conditions for future space programs. Existing TPS's for airframes of hypersonic vehicles such as ablation and reinforced carbon-carbon, however, do not fulfill all of these requirements. For example, the shuttle TPS is said to require about $40,000 \mathrm{~h}$ of maintenance between typical flights. An active TPS exploiting a counter-flow jet issuing from around a stagnation point has been considered promising since the early days of space development [1-5]. In particular, the application of a supersonic jet in the long penetration mode has been attracting a lot of attention lately in connection with drag reduction as well as thermal protection [6-8]. Nevertheless, such active systems have not yet been put into practice so far, which is in contrast to the well-matured technology of film cooling for turbine blades. The main reason is considered to lie in difficulties in the fulfillment of low mass condition, which is an obvious requirement in space missions.

(C) The Author(s). 2019 Open Access This article is distributed under the terms of the Creative Commons Attribution 4.0 International License (http://creativecommons.org/licenses/by/4.0/), which permits unrestricted use, distribution, and reproduction in any medium, provided you give appropriate credit to the original author(s) and the source, provide a link to the Creative Commons license, and indicate if changes were made. 
In the present paper we numerically investigate the performance of an active TPS for wing leading edges of hypersonic vehicles on the basis of the compressible Navier-Stokes equations. Instead of supersonic jets, we consider the application of subsonic jets, which seems advantageous in the light of low mass condition. In fact, our preliminary computations, which were carried out for the model case of a circular cylinder, indicate the achievement of high cooling effectiveness by means of a subsonic counter-flow jet; the high temperature gas coming from behind the bow shock wave formed in front of the cylinder is nearly perfectly blocked by the coolant gas issuing from around the stagnation point despite the occurrence of the Kelvin-Helmholtz instability, which enhances the mixing. These computations, however, were made with the unrealistic boundary condition that the velocity distribution of the jet at the exit was the Poiseuille-flow type for simplicity and the influence of variation of the angle of attack was not examined there. Actual cruise flights of vehicles, however, involve small but appreciable pitching motions and a subsonic counter-flow jet is expected to be easily bent upward or downward. It is also considered that the distribution of the jet at the exit must be markedly different from the Poiseuille-flow type; the Kelvin-Helmholtz instability must also have non-negligible influence on it. The assumption introduced for simplicity is removed in the present study, where an array of subsonic counter-flow jets issuing from channels embedded in the cylinder is considered.

\section{Problem}

Consider a uniform hypersonic flow of air past a circular cylinder with a radius of $120 \mathrm{~mm}$; the unit of length is millimeter in the present paper and it will be hereafter omitted unless otherwise specified explicitly. The axis of the cylinder is located at the origin $(x, y)=(0,0)$, where $x$ and $y$ are the Cartesian coordinates. A single jet (Model-1) or an array of three jets (Model-2) is issuing from a channel or three channels embedded in the cylinder. These jets are in the negative $x$ direction. The unit vector in the direction of the upstream flow is $(\cos \Omega, \sin \Omega)$; $\Omega$ corresponds to variation of the angle of attack. The coolant gas is air and the total (reservoir) temperature of the jet(s) is kept at $400 \mathrm{~K}$; the temperature of the cylinder is assumed to be the same as the total temperature of the jet(s) for simplicity. In the case of Model-1, the side-walls of the channel are located at $y= \pm 6$ (Fig. 1). In the case of Model-2, the side-walls of the lower, middle and upper channels are located at $(y=-10,-6),(y= \pm 2)$ and $(y=6,10)$, respectively (Fig. 2). We numerically analyze the flow field on the basis of the standard compressible Navier-Stokes equations for an ideal gas; no real gas effects are taken into account; the heat capacity ratio is 1.4 ; the Prandtl number is 0.72 ; and the temperature dependence of the viscosity is assumed to obey Sutherland's law. The no-slip boundary condition (for the flow velocity and the temperature) is imposed at the surface of the cylinder and the side-walls of the channel(s). The isentropic (reversible and adiabatic) condition is imposed at the inlet(s).

\section{Numerical method}

The numerical method employed in the present study is a simplified version of the shock-capturing finite volume method developed in Ref. [9]. The numerical flux 


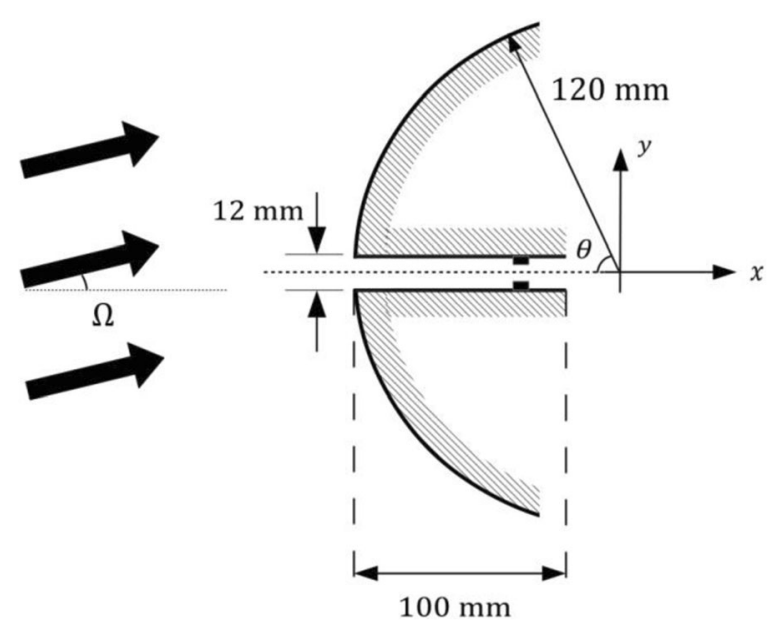

Fig. 1 Schematic of Model-1

corresponding to the Euler equations is computed as a convex combination of three parts, namely $\boldsymbol{F}^{A}, \boldsymbol{F}^{D}$ and $\boldsymbol{F}^{C}$, in the original scheme. The $\boldsymbol{F}^{A}$ is dissipative and $\boldsymbol{F}^{D}$ and $\boldsymbol{F}^{C}$ are less dissipative. The arguments of $\boldsymbol{F}^{A}$ and $\boldsymbol{F}^{D}$ are computed by MUSCL (the van-Leer slope limiter) and those of $\boldsymbol{F}^{C}$ are done by fifth order accurate Lagrange's polynomial approximation. The weights of the convex combination vary smoothly according to physical situations such that $\boldsymbol{F}^{A}, \boldsymbol{F}^{D}$ and $\boldsymbol{F}^{C}$, respectively, become dominant around shock waves, around contact discontinuities and in smooth regions. The Euler flux of the simplified scheme comprises $\boldsymbol{F}^{A}$ and $\boldsymbol{F}^{D}$; the former takes charge of regions around shocks and the latter does of those around contact discontinuities and smooth regions. The diffusive numerical flux is common for both of the schemes and is computed by the standard second order accurate central finite difference approximation; second order accuracy is considered to suffice even in the original scheme in view of the smallness of the diffusive terms, which are multiplied by the inverse of the Reynolds number. The standard RK-4 and RK-2 are employed in the original scheme and the simplified one, respectively.

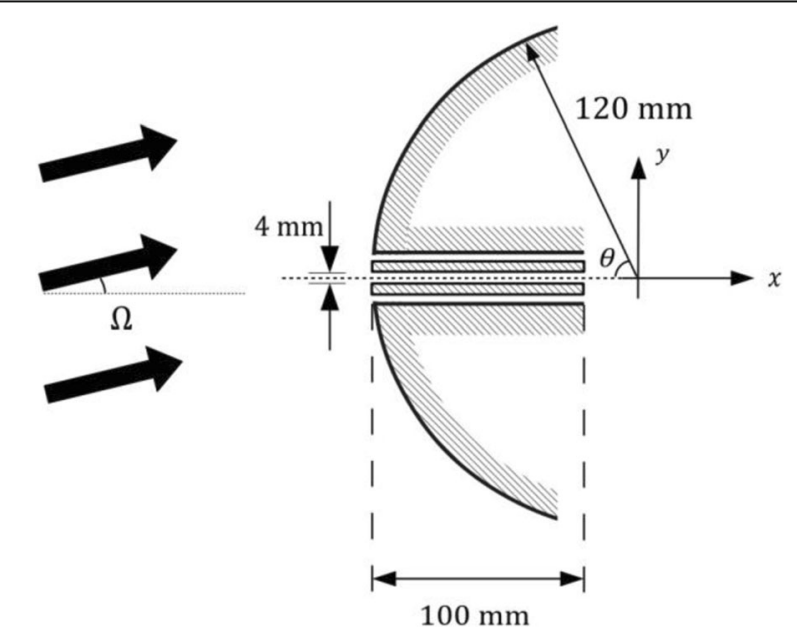

Fig. 2 Schematic of Model-2 
The robustness of the original scheme against shock anomalies such as carbuncle phenomenon and post shock oscillations is drastically enhanced by a simple procedure at the preprocessing level and its side effect appears as one-cell increase in thickness of a numerically captured shock [10]. The simplified scheme inherits the strong robustness against shock anomalies from the original scheme. The performance of the original scheme is largely comparable to that of WENO5-Rusanov, while its computational cost is $30 \sim 40 \%$ less than of that of the advanced scheme; the time consuming local characteristic decomposition is not necessary in shock capturing with MUSCL. The simplified scheme is more than 6 times as fast as the original scheme. In the present study, we put the speed of computation before the order of formal accuracy of computation, which is meaningful only if the resolution is sufficiently high.

\section{Results and discussions}

Computations were carried out for three cases of upstream condition, namely Case-A, Case-B and Case-C. Case-A corresponds to the cruising condition of the hypersonic passenger plane recently proposed by Boeing; the altitude $H$ is $30 \mathrm{~km}$ above sea level and the Mach number $\mathrm{M}_{\infty}$ is five. Case-B corresponds to the beginning of the peak heating period in typical shuttle re-entry $(H=80 \mathrm{~km}$ and $\left.\mathrm{M}_{\infty}=27\right)$ and Case- $\mathrm{C}$ does to the end of the period ( $H=50 \mathrm{~km}$ and $\left.\mathrm{M}_{\infty}=7.5\right)$. The temperature of the air behind the bow shock is estimated to be about $1400 \mathrm{~K}$, $30,000 \mathrm{~K}$ and $3300 \mathrm{~K}$ in Case-A, Case B and Case C, respectively. Real gas effects of air such as the excitation of the vibrational modes, the dissociation and the ionization are not negligibly small in Case-B and Case-C. In particular in Case-B, rarefied gas effects should also be taken into account; the Knudsen number based on the radius of the cylinder and the mean free path behind the shock is estimated to be about 0.1. Therefore the results of Case-B and Case-C, which are based on the standard compressible Navier-Stokes equations, should be regarded as just for reference. The temperature and pressure at upstream, which are denoted by $T_{\infty}$ and $P_{\infty}$, respectively, the Reynolds number $R_{\infty}$ based on the upstream condition and the diameter of the cylinder, the stagnation pressure in the case without jet(s) $P_{s t a}$ and the reservoir pressure of the jet(s) $P_{\text {res }}$ in these three cases are summarized in Table 1 . It is not easy to control the mass flow rate only by varying $P_{\text {res }}$ in the case of Model-1. For this reason, each side-wall of the channel of Model-1 equips a rectangular obstacle. These two obstacles are in the same shape and are attached to the side-walls as the components of a valve (see Fig. 3). Their size is adjusted such that the mass flow rate is nearly equal to the total mass flow rate in the case of Model-2, where no obstacles are attached to the side-walls.

Table 1 The upstream condition, the stagnation pressure and the reservoir pressure

\begin{tabular}{lllllll}
\hline & $M_{\infty}$ & $R_{\infty}$ & $T_{\infty}[K]$ & $P_{\infty}[\mathrm{Pa}]$ & $P_{\text {sta }}[\mathrm{Pa}]$ & $P_{\text {res }}[\mathrm{Pa}]$ \\
\hline Case-A & 5 & 460,000 & 230 & 1000 & $3.26 \mathrm{E}+4$ & $3.29 \mathrm{E}+4$ \\
Case-B & 27 & 2600 & 200 & 1 & $9.44 \mathrm{E}+2$ & $1.20 \mathrm{E}+3$ \\
Case-C & 7.5 & 34,000 & 270 & 76 & $5.54 \mathrm{E}+3$ & $5.85 \mathrm{E}+3$ \\
\hline
\end{tabular}




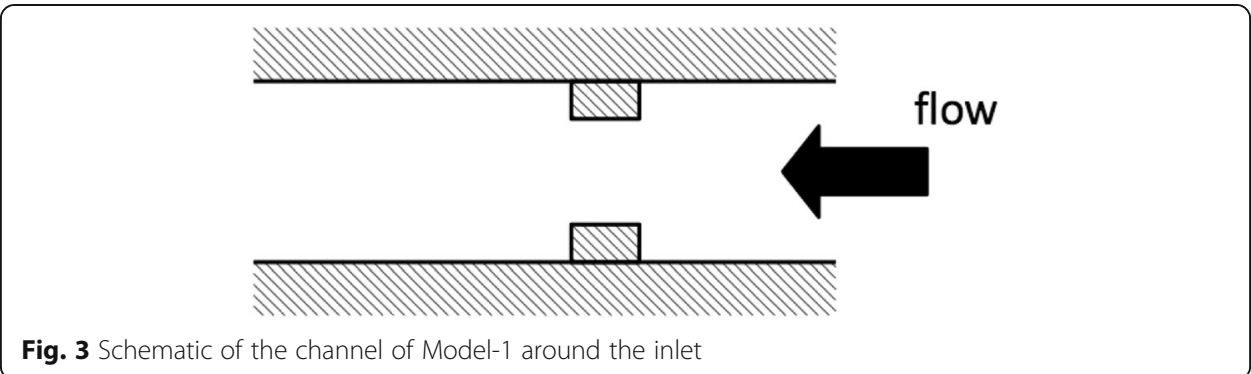

Figures 4 and 5 show results in (Case-A, Model- 1 and $\Omega=0^{\circ}$ ); a snapshot of the temperature field at a characteristic moment and the corresponding distribution of the heat flux along the cylinder surface are shown in these figures. The coolant air spreads over a wide range of the front surface of the cylinder. The Kelvin-Helmholtz instability arises around the interface of the high temperature air and the cold coolant air and the cylinder surface is intermittently exposed to the high temperature air. We quantify cooling effectiveness as $\eta$ defined by

$$
\eta=1-\frac{Q_{J}}{Q_{0}},
$$

where $Q_{J}$ and $Q_{0}$, respectively, are the integral of the heat flux over the cylinder surface in the range of $-60^{\circ}<\theta<60^{\circ}$ (see Fig. 1) for the case with jet(s) and that for

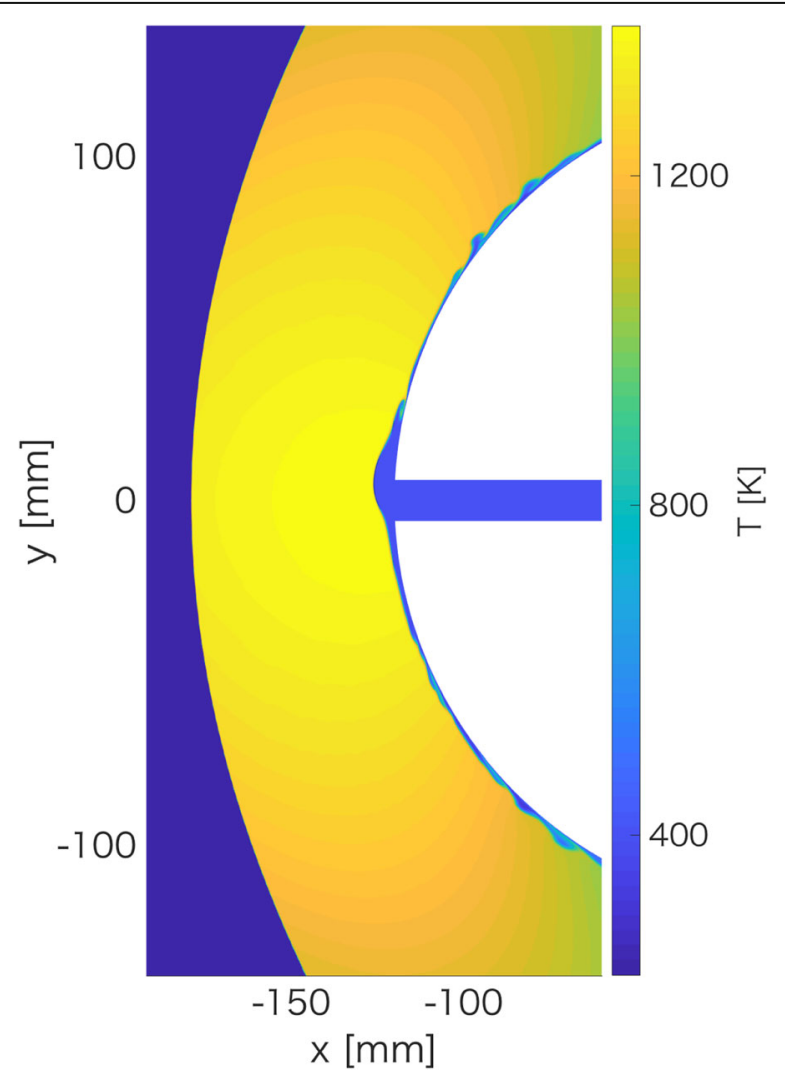

Fig. 4 A snapshot of the temperature field (Case-A, Model-1, $\Omega=0^{\circ}, 8.8 \times 10^{5}$ cells) 


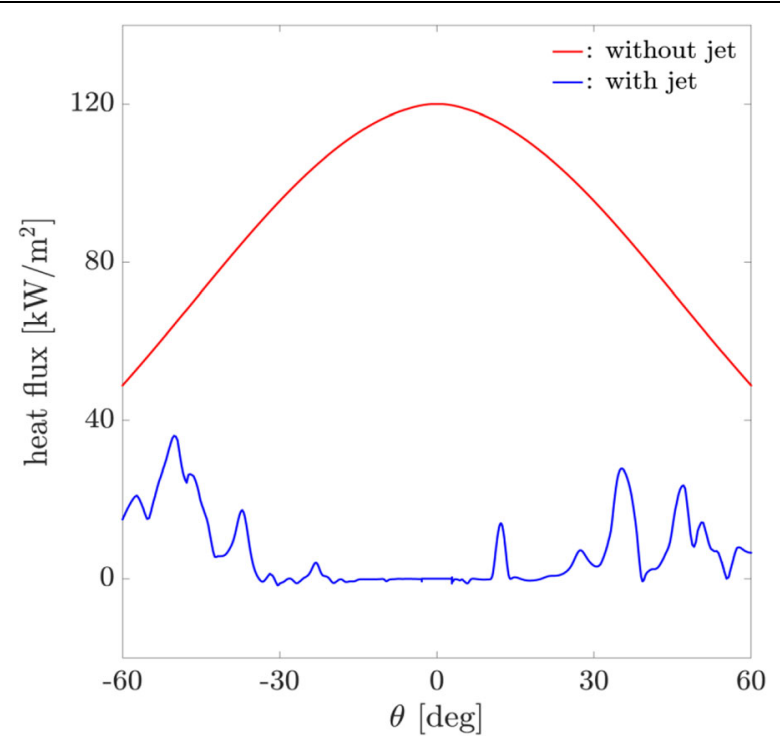

Fig. 5 The distribution of the heat flux along the cylinder corresponding to the temperature field shown in Fig. 4. The result in the case without jet (red) is also depicted for comparison

the case without jet. In the case without variation of the angle of attack $\left(\Omega=0^{\circ}\right)$, the single subsonic counter-flow jet achieves high cooling effectiveness; the time average of $\eta$ is 0.94 . As expected and mentioned before, however, it is vulnerable like smoke discharged from a chimney. Cooling effectiveness considerably decreases when the angle of attack varies even slightly. Figures 6 and 7 shows results in (Case-A, Model-1 and $\Omega=3^{\circ}$ ). Most of the time, the coolant air covers only the upper part of the cylinder surface and the lower part is directly exposed to the high temperature air. The weakness of subsonic counter-flow jet is overcome in the case of Model- 2 by sharing of the roles of three jets. Figures 8 and 9 show results in (Case-A, Model-2 and $\Omega=5^{\circ}$ ). High cooling effectiveness is achieved up to $\Omega=5^{\circ}$; the coolant air issuing from the upper and middle channels covers the upper part of the cylinder surface and that from the lower channel does the lower one. This is not due to the increase in the effective size of the exit; the distance between the upper side-wall of the upper channel and the lower side-wall of the lower channel is 20 while the height of the channel of Model-1 is 12. The cooling effectiveness is not improved by increasing the height of the channel of Model-1 to 20; the coolant air covers only the upper part of the cylinder surface as before. The time history of $\eta$ for (Case-A, Model-2 and $\Omega=5^{\circ}$ ) is shown in Fig. 10. The computations in Case-A were carried out for three cases of resolution. The number of cells is about 1 million and the minimum cell size $\Delta_{\min }$ is $3 \times 10^{-2}$ in the coarse resolution, about 2.7 million cells with $\Delta_{\min }=1.9 \times 10^{-2}$ in the fine resolution and about 5 million cells with $\Delta_{\min }=1.3 \times 10^{-2}$ in the finest resolution. In contrast to the case without jet, rather high resolution is needed in order to capture the Kelvin-Helmholtz instability, which affects cooling effectiveness; tens of thousands cells suffice for accurate prediction of the heat flux in the case without jet. More detailed structure of roll-up is captured and cooling effectiveness decreases as the resolution increases; the time average of $\eta$ is $0.94,0.92$ and 0.91 for the coarse, fine and finest resolutions, respectively. The time average of the (total) 


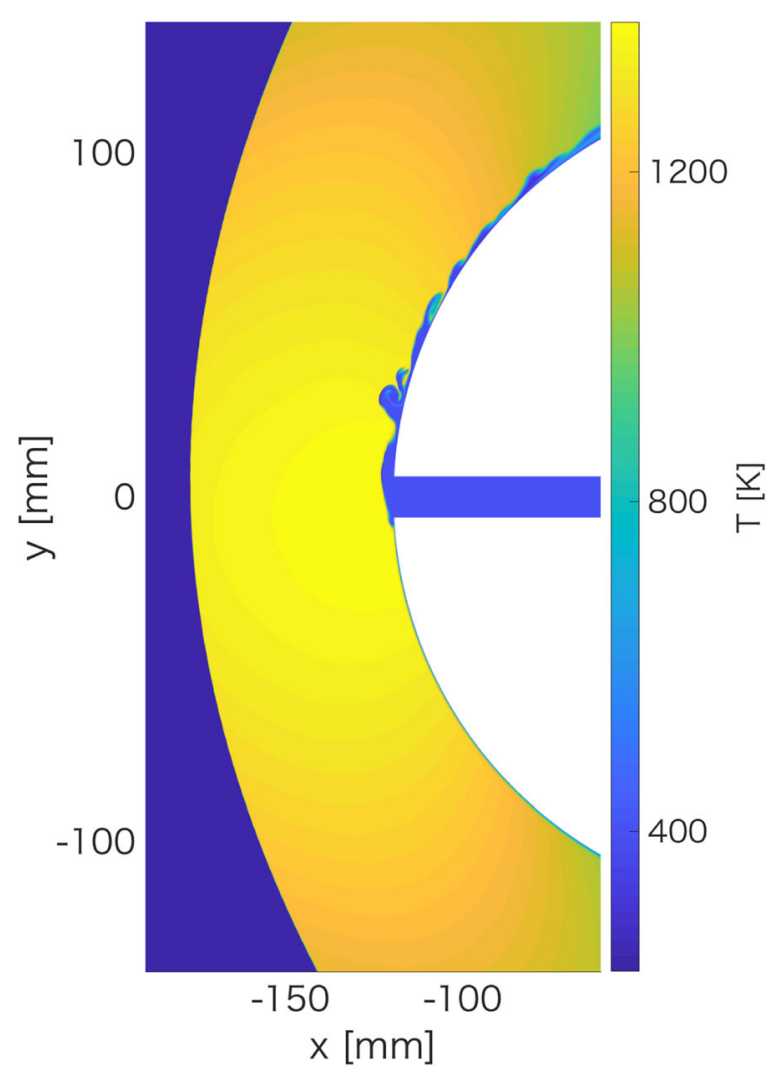

Fig. 6 A snapshot of the temperature field (Case-A, Model-1, $\Omega=3^{\circ}, 8.8 \times 10^{5}$ cells)

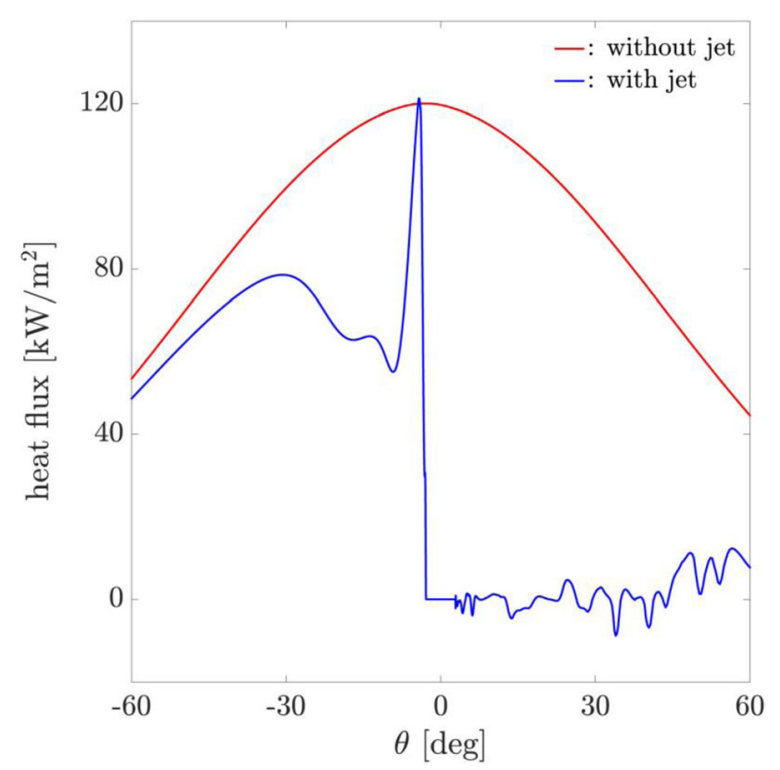

Fig. 7 The distribution of the heat flux along the cylinder corresponding to the temperature field shown in Fig. 6 


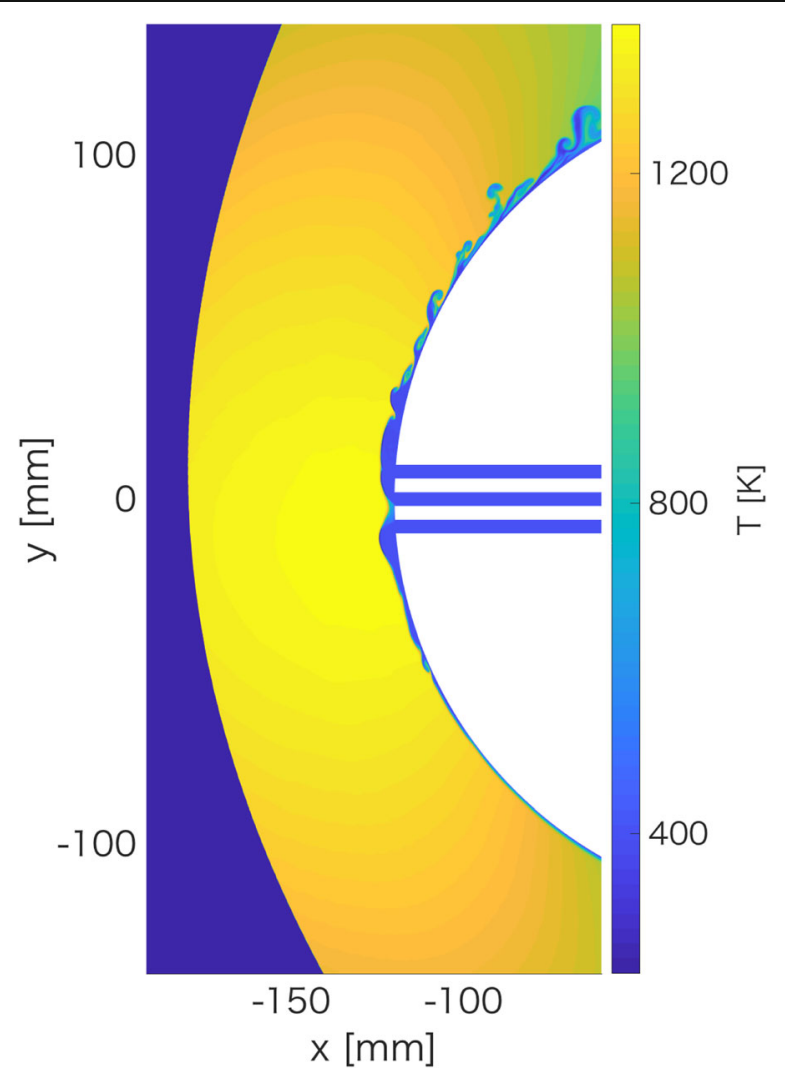

Fig. 8 A snapshot of the temperature field (Case-A, Model- $2 \Omega=5^{\circ}, 5.1 \times 10^{6}$ cells)

mass flow rate of the jet(s) is about $0.13 \mathrm{~kg} / \mathrm{ms}$, the jet Mach number is about 0.1 and the jet Reynolds number based on the jet condition and the diameter of the cylinder is about 2300 .

Figures 11 and 12 show results in (Case- $B$, Model- 2 and $\Omega=5^{\circ}$ ). The computations of Case-B were done for two cases of resolution; about 34,000 cells with $\Delta_{\min }=1.2$ in the coarse resolution and about 140,000 cells with $\Delta_{\min }=0.6$ in the fine resolution. The jet Reynolds number is about 270 and the Kelvin-Helmholtz instability does not arise. The total mass flow rate is about $0.01 \mathrm{~kg} / \mathrm{ms}$ and the jet Mach number is about 0.4. The steady state is established and $\eta$ is 0.91 in both cases of the resolution; the difference between these two numerical solutions is nearly invisible in the figures. Figures 13 and 14 show results in (Case-C, Model-2 and $\Omega=5^{\circ}$ ). The computations were done for two cases of resolution; about 190,000 cells with $\Delta_{\min }=0.4$ in the coarse resolution and about 760,000 cells with $\Delta_{\text {min }}=0.2$ in the fine resolution. The steady state is established in the case of the coarse resolution; $\eta=0.98$. In the case of the fine resolution, the coolant air intermittently rolls up around the exits of the channels, which causes the mixing of the cold coolant air and the high temperature air. The time average of $\eta$ is 0.90 , which is slightly smaller than that for the coarse resolution. The total mass flow rate is $0.04 \mathrm{~kg} / \mathrm{ms}$, the jet Mach number is about 0.2 and the jet Reynolds number is about 930 . 


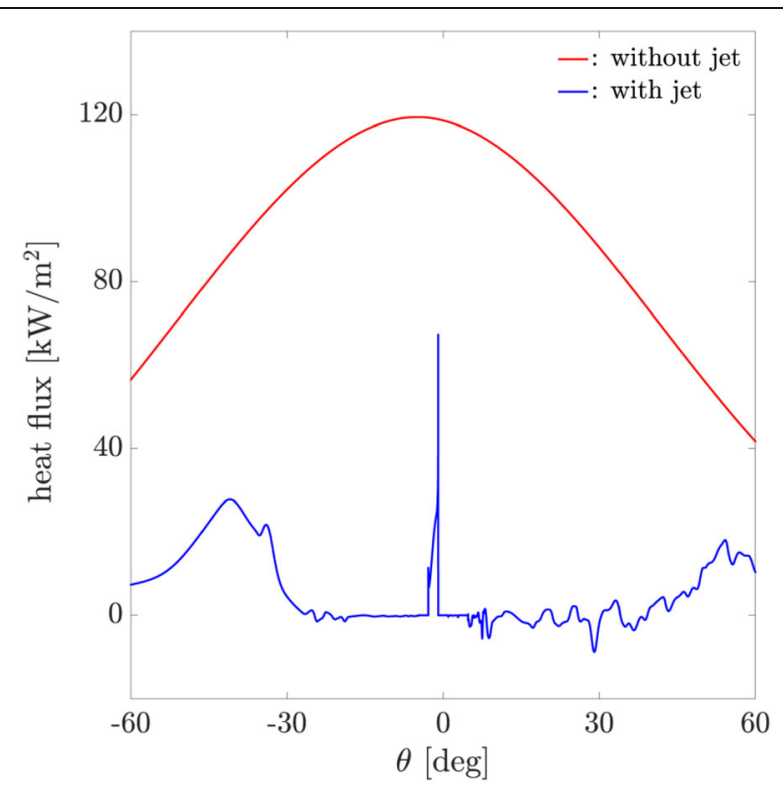

Fig. 9 The distribution of the heat flux along the cylinder corresponding to the temperature field shown in Fig. 8

The achievement of high cooling effectiveness is confirmed up to $5^{\circ}$ variation of the angle of attack in the 2D numerical computations of an array of subsonic counter-flow jets. Because the prediction of the cooling effectiveness is sensitive to the numerical behavior of the Kelvin-Helmholtz instability, more detailed numerical computations are needed. Even if the present 2D computations capture the actual flows well, it does not immediately prove the feasibility of the active TPS, however. Suppose a hypersonic vehicle with a wingspan of $20 \mathrm{~m}$ (e.g. X-30 and space shuttle orbiter). In Case-A, the active TPS consumes about $2.6 \mathrm{~kg}$ of air per second as the coolant; the total air consumed in a cruise flight of $5000 \mathrm{~km}$ amounts to about $10 \mathrm{t}$. It is not reasonable to load the vehicle with such amount of air and the suction of the outside air is

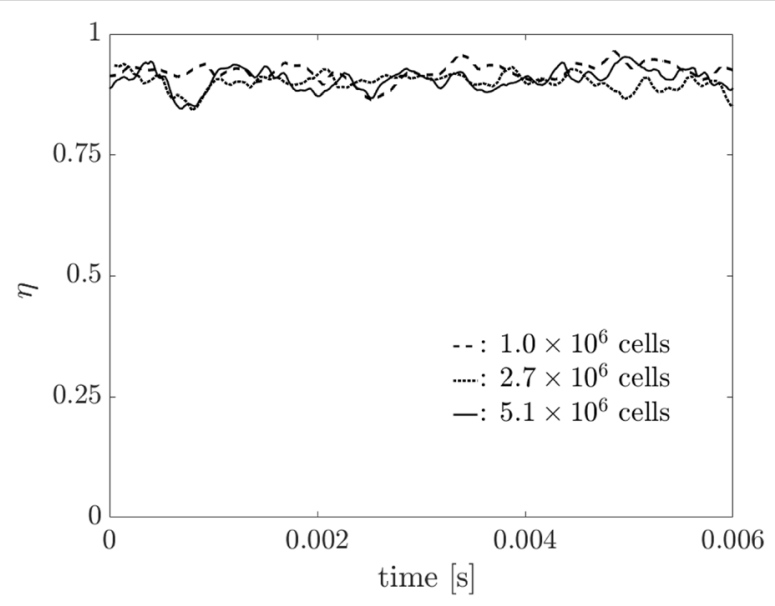

Fig. 10 The time history of $\eta\left(\right.$ Case-A, Model $\left.-2, \Omega=5^{\circ}\right)$ 


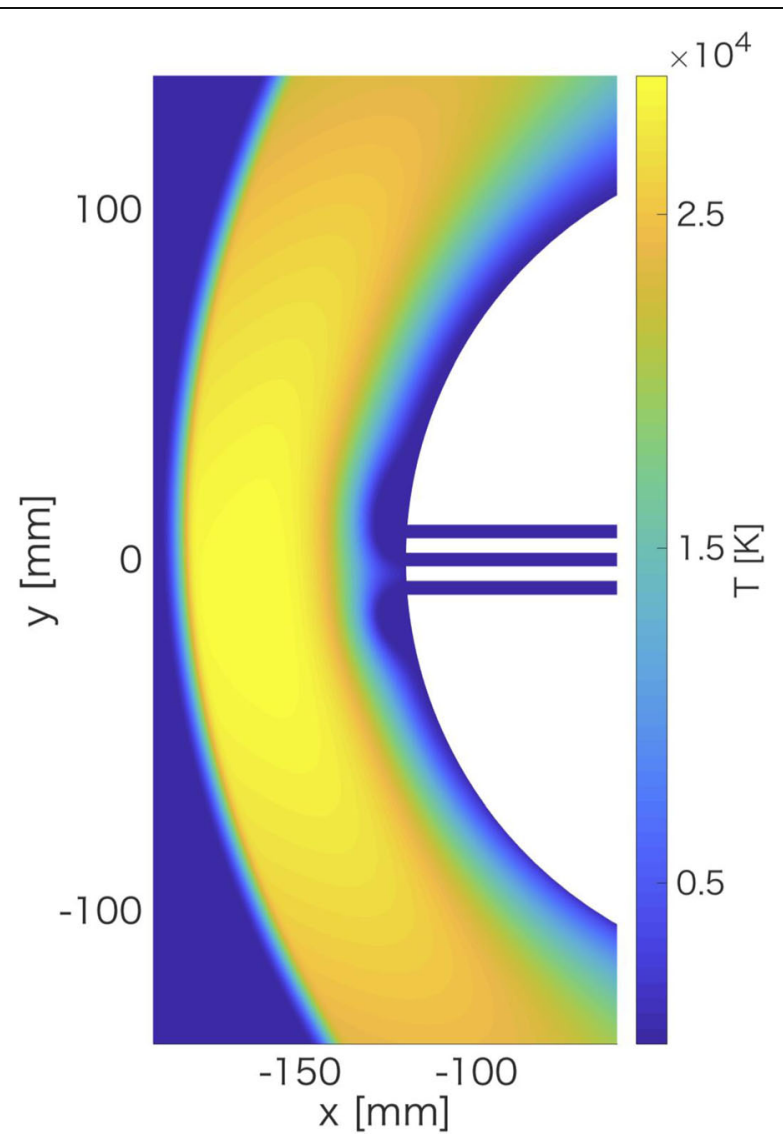

Fig. 11 A snapshot of the temperature field (Case-B, Model-2, $\Omega=5^{\circ}, 1.4 \times 10^{5}$ cells)

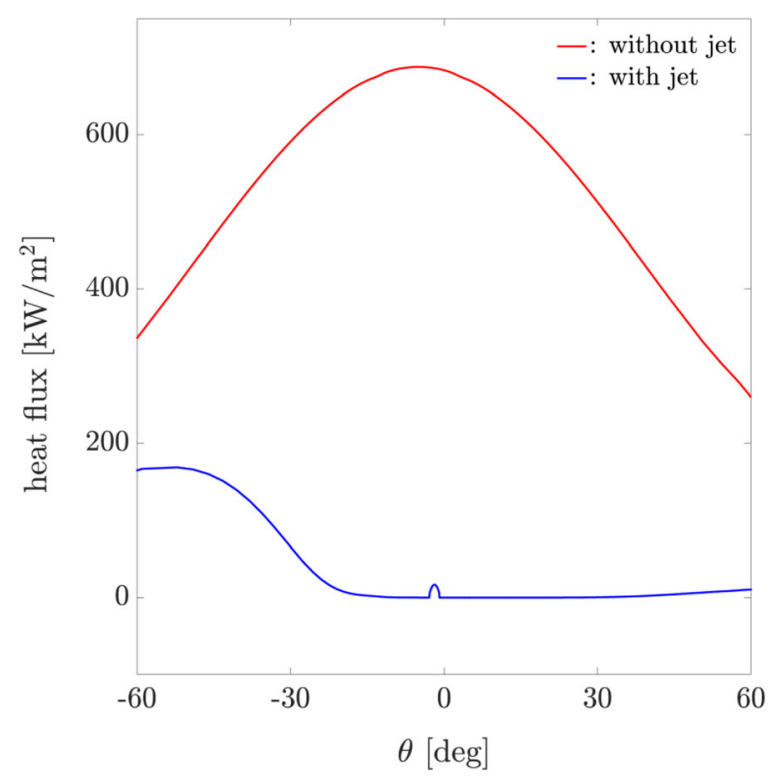

Fig. 12 The distribution of the heat flux along the cylinder corresponding to the temperature field shown in Fig. 11 


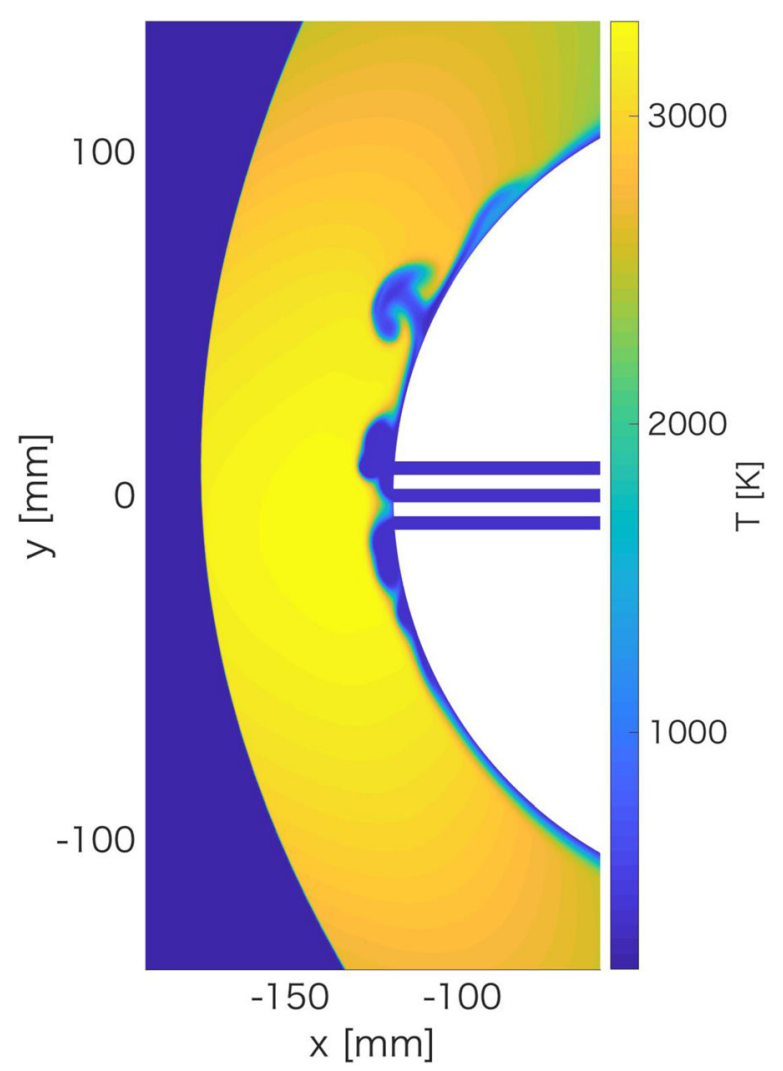

Fig. 13 A snapshot of the temperature field (Case-C, Model- $2 \Omega=5^{\circ}, 7.6 \times 10^{5}$ cells)

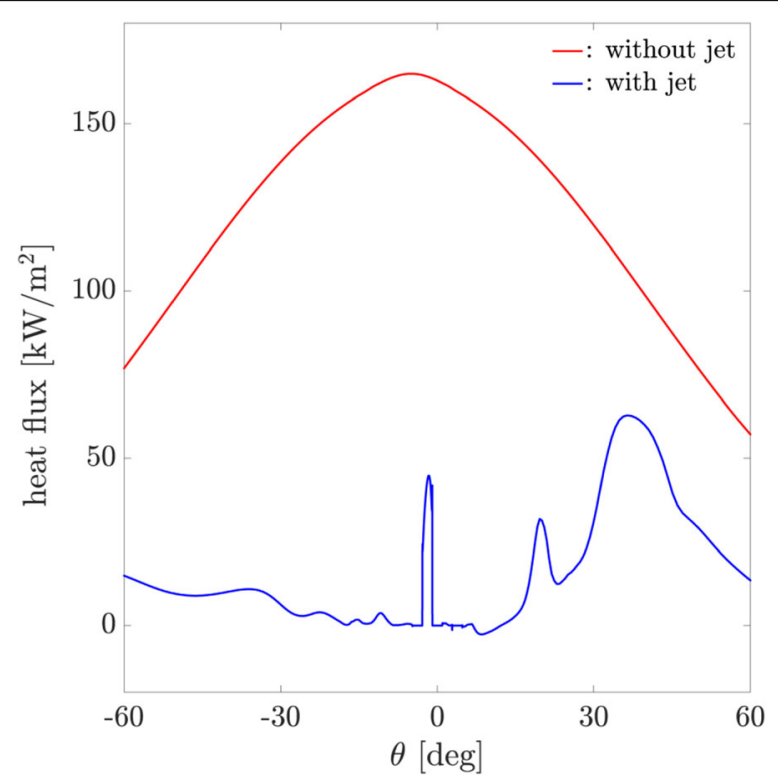

Fig. 14 The distribution of the heat flux along the cylinder corresponding to the temperature field shown in Fig. 13 
considered to be mandatory. The total temperature of the outside air, however, is about $1400 \mathrm{~K}$; the kinetic energy of molecules constituting the air at upstream, which is transformed to the internal energy during the deceleration, should be absorbed in some appropriate way. In the case of the cooling by means of water spray, where the kinetic energy is consumed as the latent heat and the coolant gas is a mixture of air and water vapor, the amount of water necessary for the flight is estimated to be about $2.9 \mathrm{t}$, which is about $2 \%$ of the gross weight of $\mathrm{X}-30$; it is reduced to $1.6 \mathrm{t}$ when the total temperature of the jets is increased to $800 \mathrm{~K}$. Similarly, it is estimated from the results of Case-B and Case-C, though just for reference, that the amount of the coolant air consumed during $800 \mathrm{~s}$ of the peak heating period in a typical shuttle re-entry is about $500 \mathrm{~kg}$, which is about $2 \%$ of the payload.

\section{Conclusions}

Subsonic jets are preferable to supersonic ones as an active TPS for hypersonic vehicles in the light of low mass condition. However, they are not robust enough against small variation of the angle of attack. The present study proposes a solution method which overcomes the weakness. It is also suggested that hypersonic passenger vehicles require another type of cooling system for the supply of the coolant. It is worth while further exploring the feasibility of active TPS's exploiting subsonic counter-flow jets. The corresponding 3D computations are now under way.

Acknowledgements

Funding from the Kyoto University Foundation is gratefully acknowledged.

Funding

The Kyoto University Foundation.

Availability of data and materials

The datasets used and/or analyzed during the current study are available from the corresponding author on reasonable request.

\section{Authors' contributions}

TO is responsible for the formulation of the problem, the analyses of numerical results and the writing. TS carried out the numerical computation and TK was engaged in the preliminary numerical computation for the present study. All authors read and approved the final manuscript.

Competing interests

The authors declare that they have no competing interests.

\section{Publisher's Note}

Springer Nature remains neutral with regard to jurisdictional claims in published maps and institutional affiliations.

Received: 11 January 2019 Accepted: 14 January 2019

Published online: 30 January 2019

\section{References}

1. Stalder JR, Inoue M (1956) A method of reducing heat transfer to blunt bodies. NACA RM A56B27a

2. Warren CHE (1959) An experimental investigation of the effect of ejecting a coolant gas at the nose of a bluff body, Royal Aircraft Establishment, Farnborought, Hants, pp 400-417

3. Swenson BL (1961) An approximate analysis of film cooling on blunt bodies by gas injection near the stagnation point. NASA TN D-861

4. Dannenberg RE (1962) Helium film cooling on a hemisphere at a Mach number of 10. NASA TN D-1550

5. Finley PJ (1966) The flow of a jet from a body opposing a supersonic free stream. J Fluid Mec 26:337-368

6. Mulmuth ND, Formin VM, Maslov AA, Fomichev VP, Shashkin AP, Korotaeva TA, Shiplyuk AN, Pozdnyakov GA (1999) Influence of a counterflow plasma jet on supersonic blunt body pressures. AIAA Paper 99-4883

7. Daso EO, Pritchett VE, Wang TS, Ota DK, Blankson IM, Auslender AH (2009) The dynamics of shock dispersion and interactions in supersonic freestreams with counterflowing jets. AIAA J 47:1313-1326 
8. Venkatachari BS, Cheng GC, Chang C-L, Zichetello B, Bilyeu DL (2013) Long penetration mode counterflowing jets for supersonic slender configurations - A Numerical study. AIAA Paper 2662

9. Ohwada T, Shibata Y, Kato T, Nakamura T (2018) A simple, robust and efficient high-order accurate shock-capturing scheme for compressible flows: towards minimalism. J Comput Phys 362:131-162

10. Ohwada T, Adachi R, Xu K, Luo J (2013) On the remedy against shock anomalies in kinetic schemes. J Comput Phys 255: $106-129$

Submit your manuscript to a SpringerOpen ${ }^{\odot}$ journal and benefit from:

- Convenient online submission

Rigorous peer review

- Open access: articles freely available online

- High visibility within the field

- Retaining the copyright to your article

Submit your next manuscript at $\boldsymbol{\nabla}$ springeropen.com 